\title{
The Effect of Chronic Otitis Externa-Media on Brainstem Auditory Evoked Potentials in Dogs
}

\section{O. BESALTI ${ }^{1}$, Y.S. SIRIN ${ }^{2}$, Z. PEKCAN ${ }^{3}$}

${ }^{1}$ Ankara University, Faculty of Veterinary Medicine, Department of Surgery, Diskapi, Ankara, Turkey

${ }^{2}$ Mayis University, Faculty of Veterinary Medicine, Department of Surgery, Samsun, Turkey

${ }^{3}$ Kirikkale University, Faculty of Veterinary Medicine, Department of Surgery, Yahsihan, Kirikkale, Turkey

Received October 18, 2007

Accepted June 11, 2008

\begin{abstract}
Besaltı O., Y.S. Sirın, Z. Pekcan: The Effect of Chronic Otitis Externa-Media on Brainstem Auditory Evoked Potentials in Dogs. Acta Vet. Brno 2008, 77: 615-624.

The objective of the study was to present normative Brainstem Auditory Evoked Potentials (BAEP) data elicited by both air-conducted clicks (30-100 dBHL) and bone-conducted clicks (100-130 dBHL) for healthy dogs, and dogs with chronic otitis externa-media (COEM). The data were analyzed to acquire reference values in 55 normal dogs and to estimate the degree of hearing impairment associated with the disease. Fifty-four dogs with COEM were divided into two degrees of severity. The first one included dogs with severe $(n=16)$, and the second with moderate COEM $(n=38)$. The recognisability wave $\mathrm{V}$ was found higher than the others at every stimulation level. Wave VII was not seen by the bone-conducted click at all stimulation levels, and also in cases with severe COEM by the air-conducted click. Recognisability of wave V was seen more than $50 \%$ at $60 \mathrm{dBHL}$ in severe COEM. This ratio was seen at $30 \mathrm{dBHL}$ in moderate COEM. In healthy dogs, the latencies of waves (I-VII) were compared between the left and the right side, and there were no significant differences between each side except for a shorter latency of wave VI. In conclusion, COEM caused increasing latency rather than total deafness, and BAEP can be suggested as an ancillary diagnostic tool in ear disorders.
\end{abstract}

Ear disease, hearing loss, BAEP, latency, canine

Chronic otitis externa is characterized by severe inflammation, with irreversible hyperplasic changes of the ear canal and the surrounding tissues, and mineralization (with subsequent ossification) of the auricular and annular cartilage (Bellah 1997). Chronic inflammation and accumulation of exudates within the canal eventually lead to the disruption of the tympanic membrane. The infection invades the middle ear (otitis media), and may eventually extend to the inner ear (otitis interna), and sometimes the facial nerve is involved (facial paralysis) (Stickney et al. 2004). Although profound hearing loss is most commonly related to neurological causes, chronic otitis externa-media (COEM) can also cause a partial hearing loss (Eger and Lindsay 1997; Krahwinkel et al. 1993). It was demonstrated that total ear canal ablation combined with bulla osteotomy, which is the standard approach to the end stage of COEM, may be the cause of a significant loss of hearing (McAnulty et al. 1995).

Deafness can be classified into conductive or sensory neural deafness according to the involved anatomical structures. Conductive hearing loss occurs with the occlusion of the ear canal, thickening or disruption of the tympanic membrane, replacement of the air within the middle ear with fluid, stiffening or damage to the ossicular chain, and loss of compliance of the vestibular or round windows (Eger and Lindsay 1997).

Brainstem Auditory Evoked Potential (BAEP) is an accurate, repeatable and noninvasive method in evaluation of the deafness in dogs and cats. It provides valuable information in the treatment protocol and prognosis, and also differentiates conductive 
and sensory neural deafness (Sims 1988). This test can be carried out by bone-conducted clicks and air-conducted clicks. By comparing the results of air and bone conduction BAEP tests, an audiologist can determine whether the hearing loss is sensory, neural, conductive or mixed. Brainstem auditory evoked potentials also provide information about which part of the anatomical structures related to hearing is affected (Cox 2002; Sims 1988).

The aim of this study was to present the effects of COEM on hearing, and provide standard values for BAEP on physiological recordings.

\section{Materials and Methods}

Fifty-five dogs of different age, sex and breed without any history or clinical signs of ear disorders, but anaesthetized for the aim of different elective surgical procedures were subjected to recordings for obtaining physiological values for the study. The cases with COEM that were diagnosed by otoscopic and radiographic examination were included prospectively. Fifty-four dogs met the criteria to be included in the study. Thirty-eight dogs had moderate COEM (stenotic canal and perforated, bulged, cloudy or opaque appearance in colour, and/ or thickened tympanic membrane) and 16 dogs had end stage COEM (ear canal narrowing or total obliteration, unable to visualize tympanic membrane). BAER were recorded under general anaesthesia which was induced using $0.05 \mathrm{mg} / \mathrm{kg}$ atropine sulphate SC (Biofarma, Turkey), $2 \mathrm{mg} \cdot \mathrm{kg}^{-1}$ xylazine hydrochlorure IM, (Alfazyne, Izmir, Turkey) and $15 \mathrm{mg} \cdot \mathrm{kg}^{-1}$ ketamine hydrochloride IM (Alfamine, Izmir, Turkey) combination. The study was approved by the Ethics Commitee of the Faculty of Veterinary Medicine, Ankara University.

Recording

BAER was recorded by Medelec Synergy 5 channel EMG/ EP device (Oxford Instrument-UK). Headset and ear tips for air-conducted clicks (Oxford, S1021) and bone conductor for bone-conducted clicks (Oxford, 017C080) were used for all cases except for dogs with a totally obliterated ear canal in which only bone conductor was used. Ear tips were inserted into both ear canals, and bone conductor was placed against the mastoid process of the temporal bone for delivering the stimulus. Subdermal stainless steel monopolar needle electrodes (Oxford, 017K103) were placed at the vertex (reference) and just rostral (active) to the base of each ear and the ground electrode was placed over the frontal sinus along the midline (Plate I, Fig.1). The impedance of the electrodes was checked to ensure that it was less than 2 kilo ohm. While stimulating one ear, the other was masked by white noise for the purpose of recording each ear individually. Impulses generated in the first 10 milliseconds, and each click was filtered (100 Hz-3 KHz). The tested ear was stimulated at $100 \mathrm{dBHL}$ to $30 \mathrm{dBHL}$ in air-conducted clicks and at $130 \mathrm{dBHL}$ to $100 \mathrm{dBHL}$ in bone-conducted clicks by the decreasing (decrement of) $10 \mathrm{dBHL}$ for each season at a click rate of $11 \mathrm{pps}$. At each level an average of 1500 clicks/stimuli were used.

Identification of the waves was carried out as reported before (Sanders and Bagley 2003; Sims 1988). Briefly, wave $\mathrm{I}$ is the first and wave $\mathrm{V}$ has a large amplitude and characteristic negative trough following the positive peak. Wave III and IV are seen either merged with or separate from each other and their amplitudes are smaller. The other waves were estimated in turn, and latencies were measured from the positive peak point of the wave.

Statistical Analysis

Latency values were compared with one-way ANOVA for healthy, severe COEM and moderate COEM cases. The latencies were analyzed in all three groups with Tukey test. The differences between right and left sides were compared using paired $t$-test.

\section{Results}

Brain stem auditory evoked responses were recorded from 110 normal ears of 55 dogs. Ages ranged from 1 to 11 years (mean 4.22). The breeds included were cross-breed $(n=30)$, Poodle $(n=6)$, German Shepherd $(n=4)$, Turkish Anatolian Sheep Dog $(n=4)$, Cocker Spaniel $(n=$ 4), Labrador $(n=3)$, Rottweiler $(n=2)$, Siberian Husky $(n=1)$ and Pointer $(n=1)$.

Moderate COEM was diagnosed in 60 ears of 38 dogs, and ages ranged from 2 to 14 years (mean 7.54). Twenty-two dogs had bilateral and 16 dogs had monolateral moderate COEM. The contralateral ears had mild inflammation or were healthy. The breeds were Cocker Spaniel $(n=11)$, Poodle $(n=8)$, German Shepherd $(n=4)$, cross breed $(n=4)$, Boxer $(\mathrm{n}=3)$, Dalmatian $(\mathrm{n}=1)$, Labrador $(\mathrm{n}=3)$, Golden Retriever $(\mathrm{n}=1)$, Turkish Anatolian Sheep Dog $(n=1)$, Pointer $(n=1)$ and Pit Bull Terrier $(n=1)$.

Severe COEM was diagnosed in 28 ears of $16 \mathrm{dogs}$, and mean ages were 11.6 years old. Twelve dogs had bilateral and 4 had monolateral severe COEM. Breed dispersion was Cocker Spaniel $(n=8)$, Poodle $(n=6)$, Labrador $(n=1)$ and Pointer $(n=1)$. Facial 

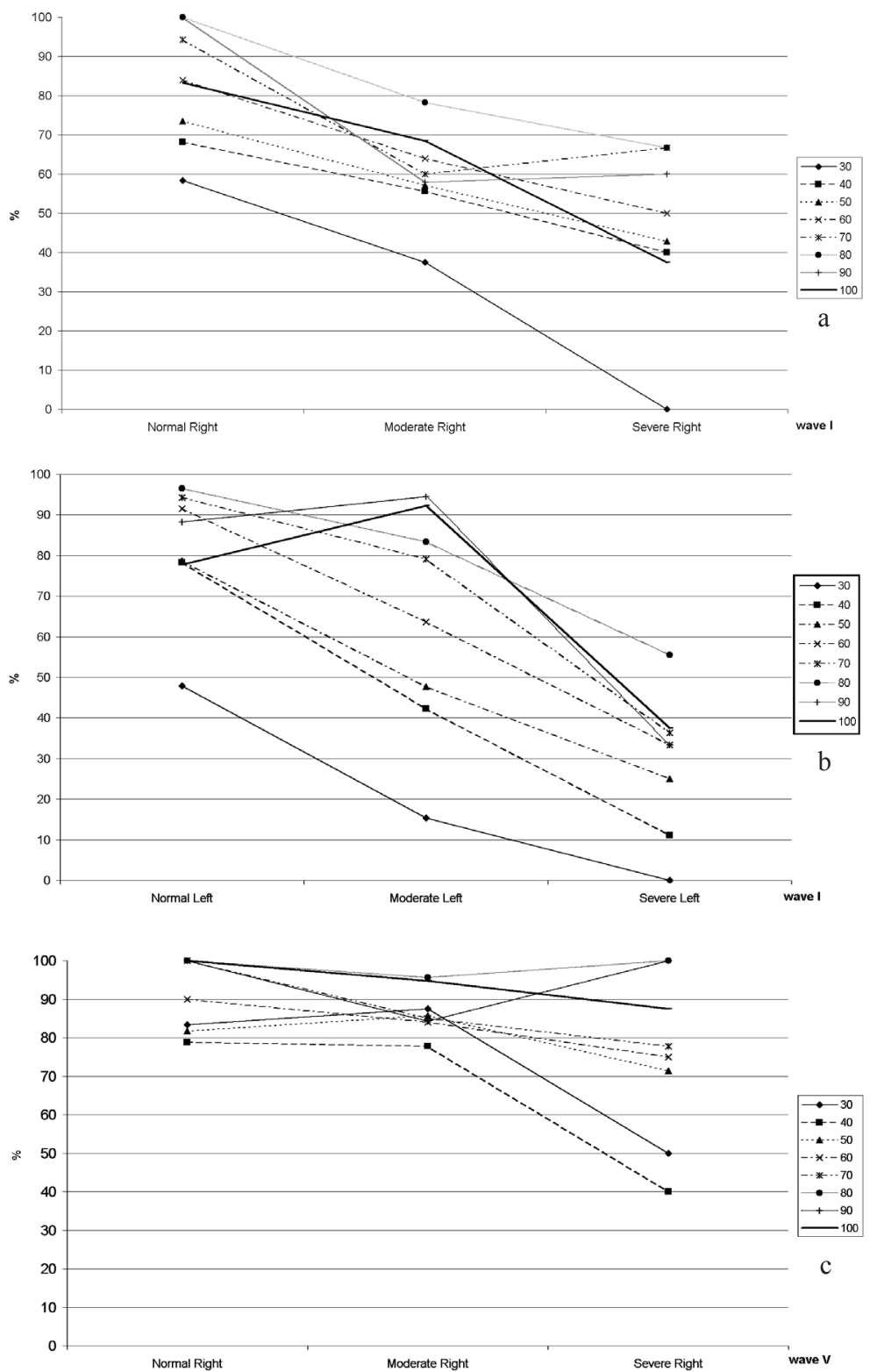

Fig. 3. The recognisability of wave I $(a, b), V(c, d)$ and VII $(e, f)$ recorded by air conducted clicks at different stimulus intensities in normal $(\mathrm{N})$, moderate $(\mathrm{M})$ and severe $(\mathrm{S})$ COEM.

paralysis was diagnosed beside severe COEM in three dogs ipsilaterally and in one dog bilaterally by neurological examination and needle EMG of the involved facial muscle.

The BAEP appears as a series of I-VII vertex positive peaks (Plate I, Fig. 2). The recognisability ratio of the waves at different stimulation levels is illustrated graphically in Figs 3a-f, 4a-f). 

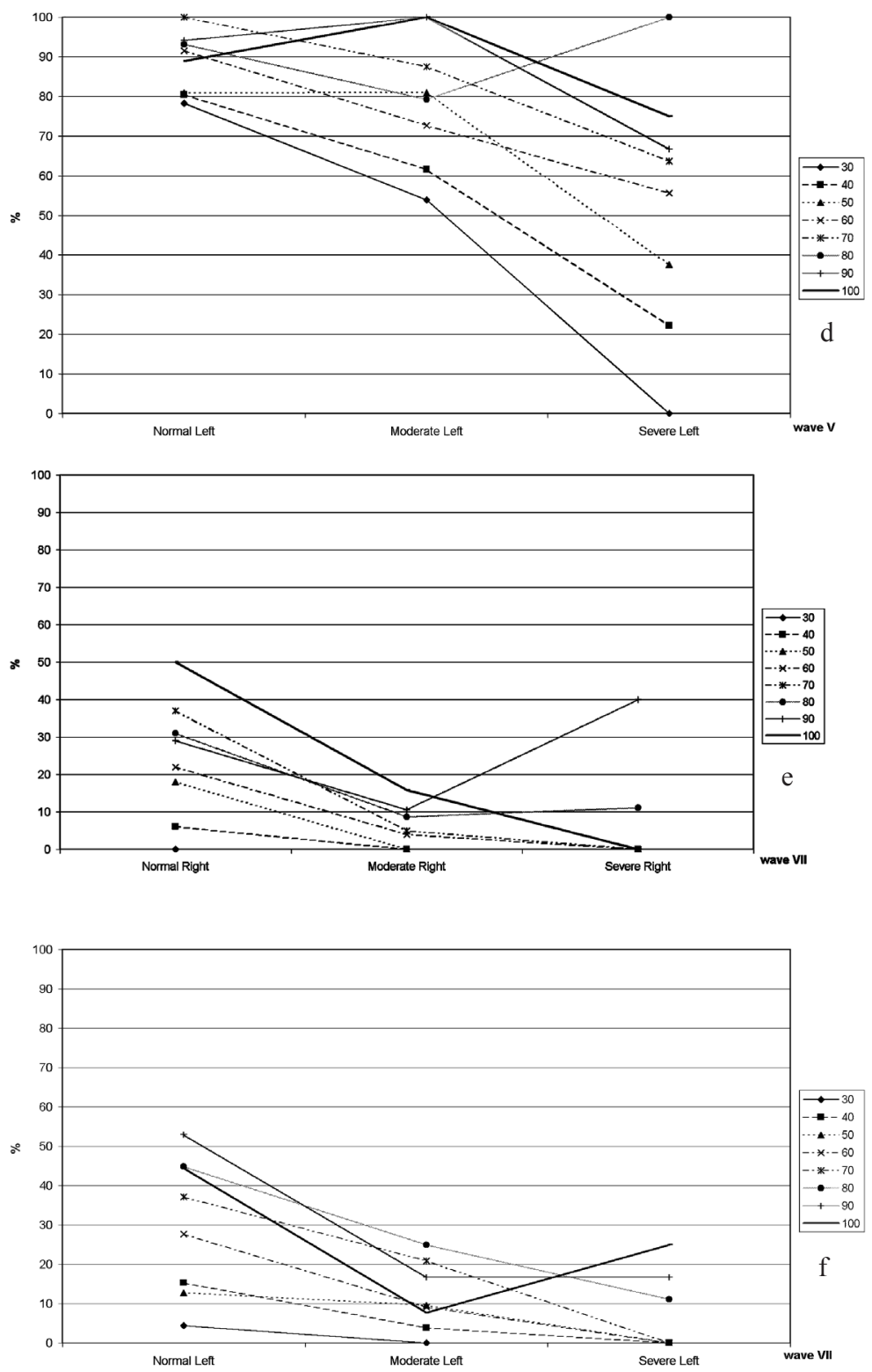

Fig. 3. The recognisability of wave I $(a, b), V(c, d)$ and VII (e, f) recorded by air conducted clicks at different stimulus intensities in normal $(\mathrm{N})$, moderate $(\mathrm{M})$ and severe $(\mathrm{S})$ COEM.

\section{Healthy dogs}

By increasing the signal intensity, the percentage recognisability of wave I, II and III increased. Wave IV appeared lower than $50 \%$ at all stimulation intensity levels. In contrast, wave V recognised at the highest amplitude at all stimulation intensity levels. Waves VI and VII were seen in a higher percentage between 50-90 dBHL. 

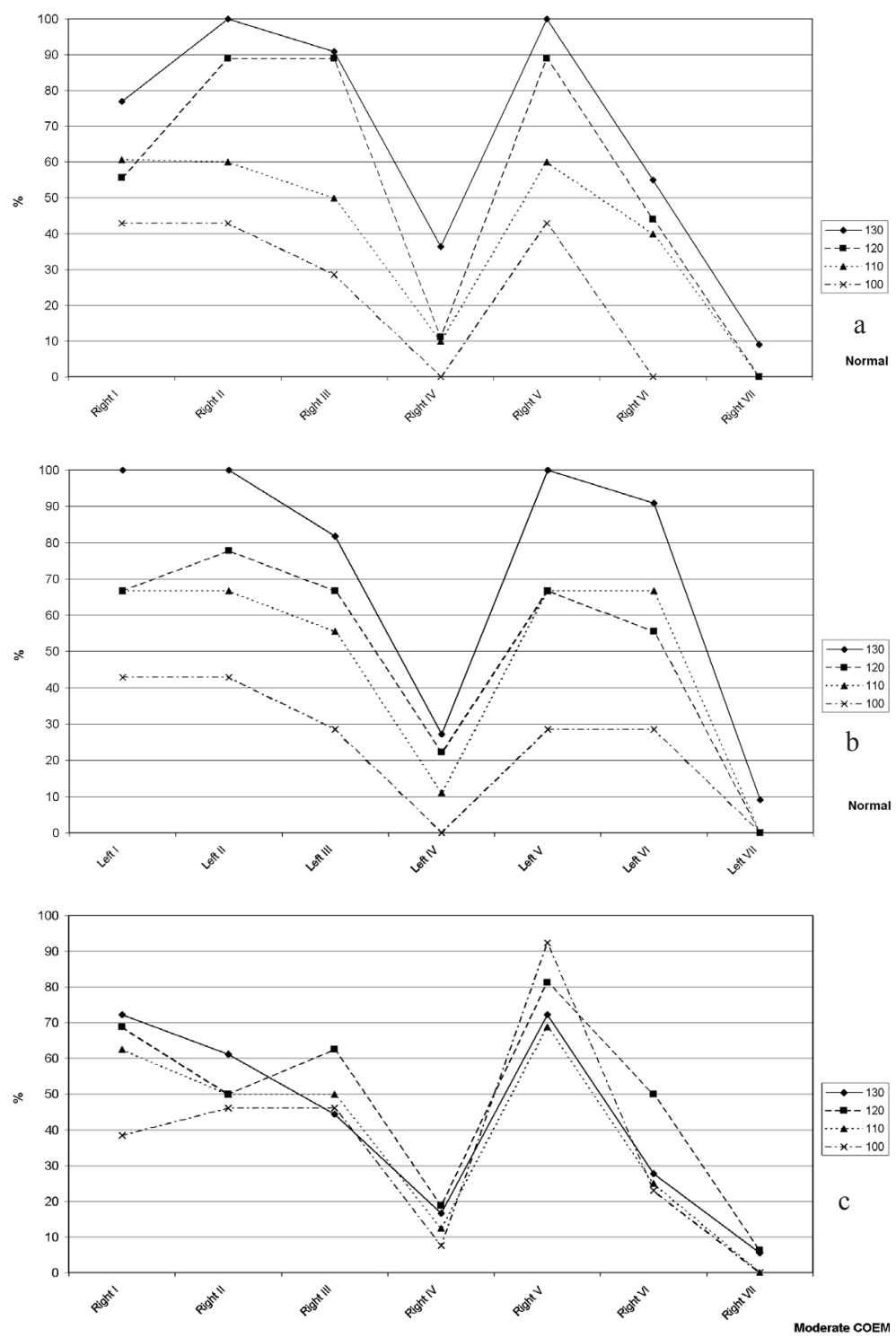

Fig. 4. The recognisability of waves elicited by bone conducted clicks at different stimulus intensities in healthy (a, b), moderate COEM (c, d), severe $\operatorname{COEM~(e,~f)~cases.~}$

The recognisability ratio of the waves in moderate COEM: At $30 \mathrm{dBHL}$ wave IV and VII, at $40 \mathrm{dBHL}$ wave VII and at $50 \mathrm{dBHL}$ wave IV and VII, and at 60, 70 and $100 \mathrm{dBHL}$ wave VII did not appear. The recognisability ratio of the waves of moderate COEM; wave IV and VII at 50 and $60 \mathrm{dBHL}$ were zero, and at 70-90 dBHL wave VII was not seen. Wave VII was not seen in bone-conducted clicks records at any stimulation intensity level.

The latency values for both air-conducted clicks and bone-conducted clicks are presented in Tables 1 and 2. Significant difference at $70 \mathrm{dBHL}$ of air-conducted click records was seen as: The latency of wave I and II, III in healthy ears was smaller than in moderate 

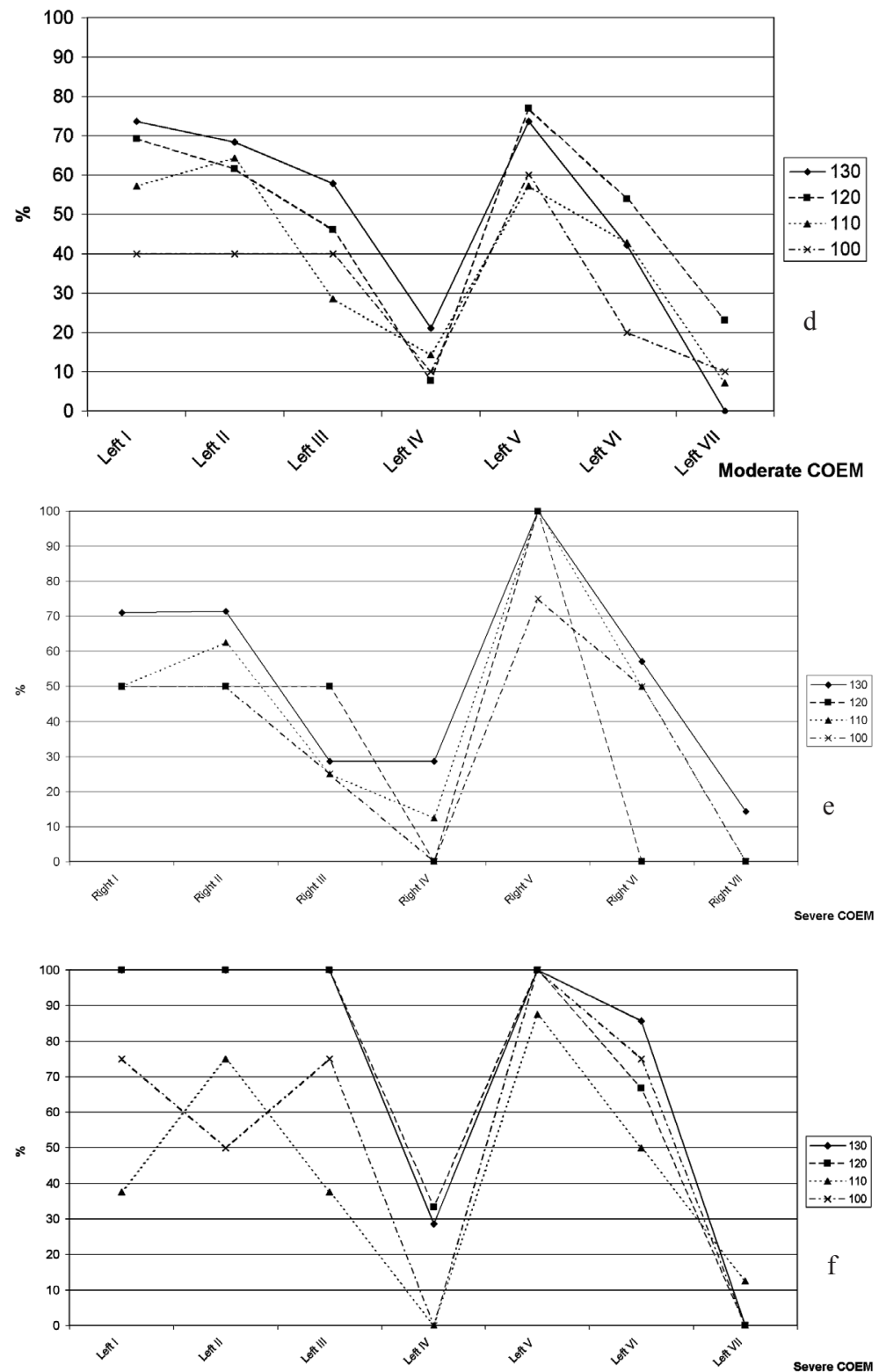

Fig. 4. The recognisability of waves elicited by bone conducted clicks at different stimulus intensities in healthy $(a, b)$, moderate $\operatorname{COEM}(\mathrm{c}, \mathrm{d})$, severe $\operatorname{COEM}(\mathrm{e}, \mathrm{f})$ cases.

and/or severe COEM. The latency of wave V (on the left side) and VI (on the right side) in healthy ears was smaller than in severe COEM. When the latency values were compared regarding the left or right side, there were no significant differences except for wave VI which was smaller on the left side than on the right.

Significant differences of latency at $110 \mathrm{dBHL}$ of bone-conducted click records were seen 


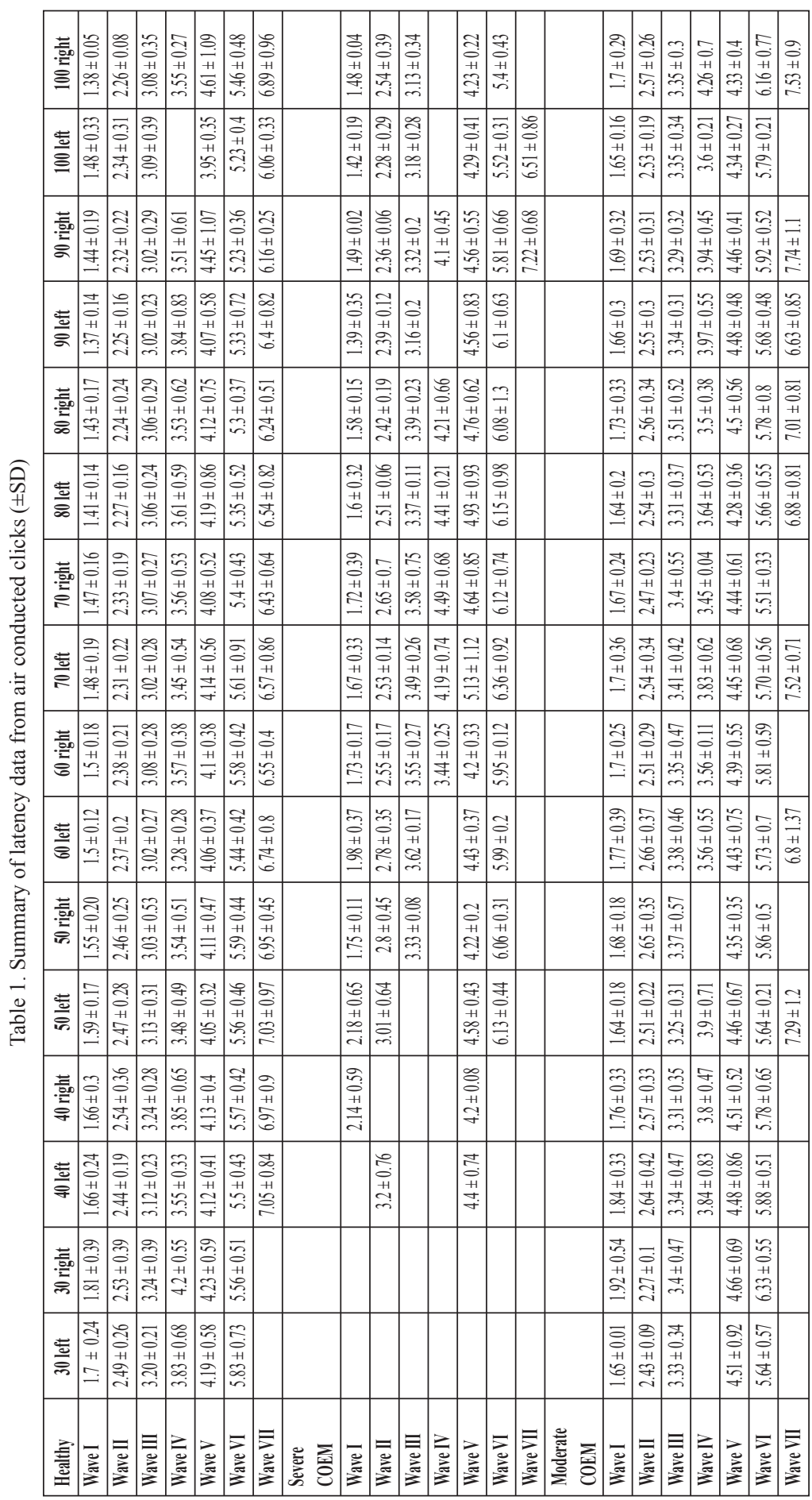




\begin{tabular}{|c|c|c|c|c|c|c|c|c|c|c|c|c|c|c|c|c|c|c|c|c|c|}
\hline 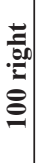 & $\begin{array}{l}\stackrel{2}{0} \\
\dot{0} \\
+1 \\
\infty \\
\vec{i} \\
\text { i. }\end{array}$ & $\begin{array}{l}= \\
0 \\
0 \\
+1 \\
= \\
\dot{=}\end{array}$ & 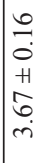 & & $\begin{array}{l}\tilde{\partial} \\
\tilde{o} \\
+ \\
\tilde{\sigma} \\
\text { in }\end{array}$ & & & & $\begin{array}{l}\infty \\
? \\
0 \\
H \\
0 \\
\dot{v} \\
\text { i. }\end{array}$ & $\begin{array}{c}n \\
? \\
0 \\
+1 \\
\infty \\
0 \\
i\end{array}$ & & & 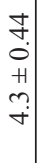 & $\left|\begin{array}{c}0 \\
0 \\
0 \\
+1 \\
\overline{0} \\
\dot{n}\end{array}\right|$ & & $\begin{array}{c}= \\
0 \\
0 \\
+1 \\
\tilde{0} \\
\sim \\
\sim\end{array}$ & 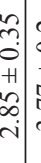 & 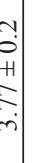 & 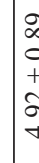 & $\begin{array}{lll}0 \\
+ \\
\end{array}$ & \\
\hline $\begin{array}{l}\frac{\vec{t}}{\overrightarrow{0}} \\
\stackrel{\Xi}{\Xi}\end{array}$ & $\begin{array}{l}\infty \\
\stackrel{0}{\circ} \\
+ \\
= \\
\vec{i}\end{array}$ & $\begin{array}{l}= \\
\overline{0} \\
+1 \\
\stackrel{n}{2} \\
\dot{m}\end{array}$ & 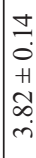 & & 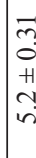 & $\begin{array}{l}\infty \\
0 \\
+ \\
\infty \\
0\end{array}$ & & & 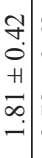 & 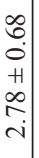 & 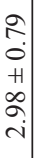 & $\begin{array}{l}\bar{m} \\
0 \\
+ \\
\hat{\sigma} \\
\dot{m}\end{array}$ & $\left|\begin{array}{l}n \\
+ \\
0 \\
+1 \\
\hat{n} \\
n \\
n \\
n\end{array}\right|$ & & & $\left|\begin{array}{c}n \\
n \\
0 \\
+1 \\
o \\
o \\
i\end{array}\right|$ & 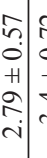 & 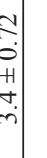 & $\mid \begin{array}{c}\mathfrak{z} \\
0 \\
+ \\
3 \\
\gamma\end{array}$ & $\begin{array}{l}8 \\
8 \\
0\end{array}$ & \\
\hline 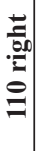 & 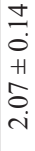 & $\begin{array}{l}\sigma \\
\partial \\
0 \\
+1 \\
\tilde{\sigma} \\
\dot{r}\end{array}$ & $\begin{array}{l}\tilde{m} \\
\text { on } \\
+1 \\
\stackrel{2}{n} \\
m\end{array}$ & & 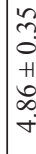 & $\begin{array}{l}2 \\
0 \\
0 \\
+ \\
\frac{2}{2}\end{array}$ & & & 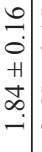 & $\begin{array}{l}n \\
0 \\
0 \\
+ \\
b \\
b \\
i\end{array}$ & $\begin{array}{l}\hat{N} \\
0 \\
+ \\
n \\
\tilde{n} \\
\end{array}$ & & $\begin{array}{l}\infty \\
+ \\
+ \\
+ \\
+1 \\
\infty \\
\stackrel{1}{0} \\
+ \\
+\end{array}$ & 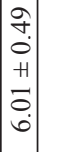 & & 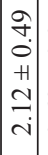 & $\begin{array}{c}c \\
\vdots \\
+1 \\
+ \\
\vdots \\
i \\
i\end{array}$ & 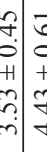 & 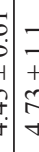 & $\begin{array}{ll}0 \\
0 \\
0\end{array}$ & \\
\hline$\frac{\stackrel{ \pm}{\frac{\omega}{\sigma}}}{\underline{G}}$ & $\begin{array}{l}m \\
\stackrel{m}{0} \\
+ \\
\hat{0} \\
\dot{i}\end{array}$ & 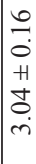 & 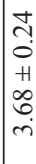 & & $\begin{array}{l}n \\
0 \\
0 \\
+1 \\
\text { on } \\
\text { in }\end{array}$ & $\begin{array}{l}c \\
0 \\
+ \\
n \\
2\end{array}$ & & & 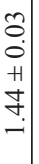 & $\begin{array}{l}m \\
3 \\
0 \\
+ \\
+ \\
\\
i\end{array}$ & $\begin{array}{c}\tilde{f} \\
0 \\
+1 \\
\tilde{n} \\
n \\
r\end{array}$ & & 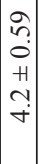 & $\left|\begin{array}{c}0 \\
0 \\
0 \\
H \\
n \\
n \\
n \\
n\end{array}\right|$ & & $\begin{array}{c}2 \\
\hat{0} \\
+1 \\
\tilde{\infty} \\
- \\
-\end{array}$ & 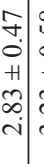 & 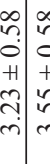 & 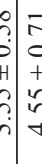 & $\begin{array}{l}8 \\
0 \\
8 \\
8 \\
2 \\
\text { in }\end{array}$ & \\
\hline 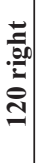 & 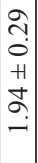 & $\begin{array}{l}+ \\
\stackrel{0}{0} \\
\ddot{H} \\
\dot{\infty} \\
i\end{array}$ & $\begin{array}{l}\text { J } \\
0 \\
+1 \\
\tilde{n} \\
n \\
\text { r. }\end{array}$ & & $\begin{array}{l}\vec{\sigma} \\
\text { o } \\
+ \\
\dot{\gamma} \\
\dot{\gamma}\end{array}$ & $\begin{array}{l}2 \\
2 \\
+ \\
\\
2\end{array}$ & & & & & & & $\begin{array}{c}\vec{n} \\
0 \\
+ \\
\infty \\
\infty \\
\sim \\
+\end{array}$ & & & $\begin{array}{c}\overline{\tilde{c}} \\
0 \\
+1 \\
\tilde{\infty} \\
- \\
-\end{array}$ & 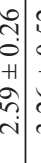 & 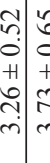 & 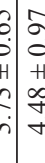 & & \\
\hline 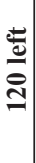 & 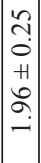 & 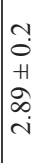 & 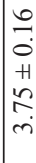 & $\begin{array}{l}+ \\
0 \\
0 \\
+1 \\
m \\
\ddot{+}\end{array}$ & 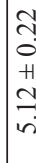 & 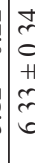 & & & 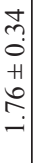 & 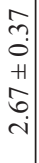 & $\begin{array}{c}\infty \\
\tilde{0} \\
0 \\
+1 \\
\sim \\
\tilde{n}\end{array}$ & & 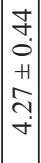 & $\left|\begin{array}{c}n \\
n \\
0 \\
+1 \\
m \\
\hdashline \\
\end{array}\right|$ & & $\begin{array}{c}+ \\
0 \\
+ \\
\dot{0} \\
\infty \\
- \\
-\end{array}$ & 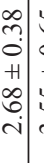 & 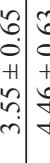 & 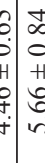 & & \\
\hline 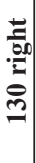 & 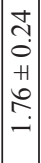 & 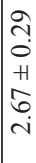 & 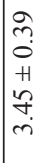 & $\begin{array}{l}\stackrel{2}{\sigma} \\
\dot{0} \\
+1 \\
\hat{\alpha} \\
\dot{m}\end{array}$ & $\begin{array}{l}8 \\
0 \\
0 \\
+1 \\
n \\
+ \\
+\end{array}$ & $\begin{array}{l}2 \\
0 \\
+\infty \\
\infty\end{array}$ & & & 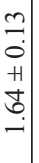 & 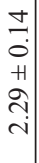 & $\begin{array}{l}+ \\
\dot{0} \\
+ \\
\dot{0} \\
\infty \\
ن \\
\dot{\sim}\end{array}$ & $\begin{array}{l}\dot{J} \\
? \\
0 \\
+ \\
\dot{H} \\
\dot{r}\end{array}$ & 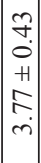 & $\left|\begin{array}{c}2 \\
2 \\
0 \\
+1 \\
\infty \\
\tilde{m} \\
i n\end{array}\right|$ & & $\begin{array}{c}0 \\
0 \\
+1 \\
\infty \\
\\
-\end{array}$ & 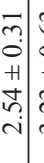 & 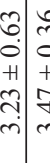 & 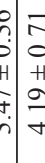 & 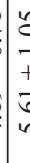 & \\
\hline 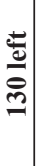 & $\begin{array}{c}0 \\
1 \\
0 \\
+1 \\
\infty \\
\infty \\
\infty \\
-\end{array}$ & $\mid \begin{array}{l}\hat{n} \\
0 \\
+ \\
\tilde{N} \\
\hat{i}\end{array}$ & 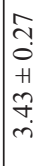 & 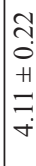 & $\begin{array}{l}0 \\
n \\
0 \\
+1 \\
0 \\
\dot{0} \\
+\end{array}$ & $\begin{array}{l}7 \\
7 \\
+ \\
z \\
\text { D }\end{array}$ & & & 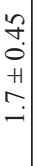 & 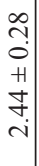 & 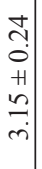 & 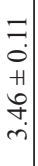 & 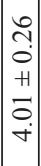 & $\left|\begin{array}{c}\infty \\
n \\
0 \\
+ \\
+ \\
6 \\
\dot{n}\end{array}\right|$ & & $\begin{array}{l}0 \\
+ \\
0 \\
+1 \\
i \\
\hat{-} \\
-1\end{array}$ & 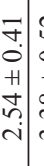 & 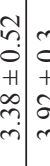 & 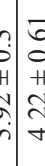 & & \\
\hline 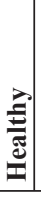 & $\bar{z}$ & 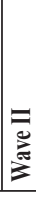 & $\underline{n}$ & 吾 & 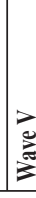 & 13 & 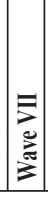 & 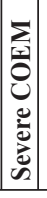 & 总 & 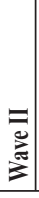 & 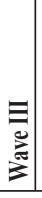 & 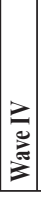 & \begin{tabular}{l}
$\overrightarrow{0}$ \\
0 \\
0 \\
\hdashline
\end{tabular} & 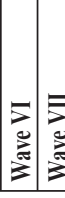 & 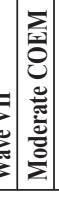 & 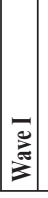 & 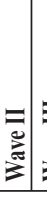 & 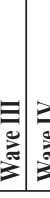 & 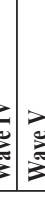 & & $\Rightarrow 3$ \\
\hline
\end{tabular}

as follows: Wave I was lower in severe COEM than in healthy ears; however, severe COEM values were lower than moderate COEM on the left side. Normal values of wave II were higher than in moderate and severe chronic COEM. Wave $\mathrm{V}$ was smaller in healthy ears than in severe COEM.

\section{Discussion}

Dogs that only have normal traces at 40 dBHL or above, have a mild hearing loss, whereas those with normal traces at 60 $\mathrm{dBHL}$ and above are considered to have a moderate hearing loss. Dogs with normal traces at 75 dBHL or above have a severe or profound hearing loss (Cox 2002). According to this classification there was no hearing problem in 55 normal dogs in which BAER was recognized at 30 dBHL. In addition, recognis ability of wave $\mathrm{V}$ at all stimulus intensities in a higher degree means that it can be used for determining the threshold, as reported before (Cox 2002; Sims 1988). In this study, severe COEM cases had moderate hearing problems. In these 
cases the recognisability ratio of wave $\mathrm{V}$ with more than $50 \%$ was seen at $60 \mathrm{dBHL}$ intensity. In moderate COEM there was a non-significant hearing loss, from which it was deducted that the recognisability rate was more than $50 \%$ at $30 \mathrm{dBHL}$.

The presence of wave VII whose generator is uncertain in healthy dogs and its absence in severe COEM cases was found interesting. As mentioned before for these cases, having higher hearing thresholds and the absence of wave VII seem to be related. However, before making a clear conclusion, more cases with severe COEM should be investigated. Another interesting finding related to wave VII was its absence at all stimulus intensities in boneconducted click records. Another conclusion for wave VII can be deducted as conductive structure related to the hearing having a role in the generation of this wave. Since the generator of wave VII is an auditory reflexion (Sims 1988, 2003), in severe COEM cases this reflexion is prevented.

The latency of all waves increased with the severity of otitis, although the prolongation of times was only significant in advanced cases (Eger and Lindsay 1997). In BAER evoked by air-conducted clicks, latency of wave I increased in both moderate and severe COEM cases compared to healthy dogs in our study. For wave II on the left side, and for wave III on both sides, for wave $\mathrm{V}$ which was recognized at approximately all stimulus intensities, the latency was increased in both moderate and severe COEM compared to healthy dogs. These findings are similar to Eger and Lindsay's report (1997).

The sound waves from the external ear canal are transmitted through the tympanic membrane through the ossicles, which articulate to amplify sound and transmit vibrations to the fluid-filled inner ear. On the surface of the organ of Corti, the inner and outer hair cells lie that connect with the spiral ganglion for transmission to the cochlear nerve (Cook 2004). There is a general agreement that wave I of the BAEP is generated by acoustic nerve activity (Sims 1988). Significant differences of latency, which was lower in severe COEM than normal wave I at $110 \mathrm{dBHL}$ of bone-conducted click can be explained as follows: In severe COEM cases ear canals are mostly obstructed due to chronic inflammation, and sound waves can not be transmitted through the external ear canal. By the bone-conducted click ear canal, tympanic membrane, and perhaps the ossicles vibrations are bypassed, and the receptors in the organ of Corti detect this vibration and the acoustic nerve is stimulated by this impulse. This is the possible cause of the decreased latency of wave I of BAEP in severe COEM.

In a study of BAEP in 58 healthy dogs, 4-5 vertex positive waves were explained and latency values at $60 \mathrm{dBHL}$ were I: $1.49 \pm 0.13 \mathrm{~ms}$; II: $2.32 \pm 0.14 \mathrm{~ms}$; III: $3.01 \pm 0.25 \mathrm{~ms}$; IV: $4.22 \pm 0.27 \mathrm{~ms}$ and V: $5.55 \pm 0.37 \mathrm{~ms}$ (Bodenhamer et al. 1985). In our study 7 vertex positive waves were identified at different recognisability rates, and also different latency values. The differences are related to the different explanation of wave V.

In conclusion, reference values for both latency and recognisability rate for BAEP in healthy dogs were obtained. Chronic otitis externa-media causes an increase in latency rather than total deafness. Even in severe COEM, dogs have the ability to hear, but to a lesser extent than healthy ones. BAER may be suggested as an ancillary diagnostic tool in ear disorders. Sensory neural deafness associated with COEM can also be differentiated by this test.

\section{Vliv chronické otitis externa-media na akustickou odpověd' mozkového kmene u psů}

Cílem studie bylo prezentovat normativní hodnoty akustické odpovědi mozkového kmene (BAEP) na zvukový podnět (kliknutí), šíření zvukové vlny vzduchem (30-100dBHL) a její převod sluchovými kůstkami (100-130 dBHL) u zdravých psů a u psů s chronickou otitis externa-media (COEM). Analýza dat u 55 zdravých psů byla provedena za účelem získání referenčních hodnot pro odhad míry zhoršení sluchu provázející chronický zánět vnějšího a středního ucha u psa. Psi postižení COEM byli rozděleni do dvou skupin dle zá- 
važnosti onemocnění. Do první skupiny byli zahrnuti jedinci s vážnou formou onemocnění $(n=16)$, druhá skupina obsahovala jedince s mírným stupněm COEM $(n=38)$. Ve všech úrovních podráždění byl patrný vzrưst vlny $\mathrm{V}$ ve srovnání s ostatními vlnami. U převodu zvukové vlny sluchovými kůstkami chyběla úplně vlna VII ve všech stimulačních hladinách a $\mathrm{v}$ případě těžkého zánětu chyběla vlna VII i u přenosu akustického signálu vzduchem. Vlna $\mathrm{V}$ byla patrná ve více než $50 \%$ prŕpadů na hladině $60 \mathrm{dBHL}$ u jedinců v první skupině. Stejný procentuální podíl byl na hladině $30 \mathrm{dBHL}$ pozorován u mírné COEM. U zdravých psů byla latence jednotlivých vln (I - VII) na pravé i levé straně srovnatelná, hodnoty na pravé i levé straně se statisticky významně nelišily s výjimkou menšího opoždění v př́ípadě vlny VI. Na závěr je možno konstatovat, že COEM způsobuje spíše vyšší latenci v převodu zvuku než ztrátu sluchu a BAEP lze využít jako jednu z pomocných metod $\mathrm{v}$ diagnostice sluchových poruch.

Acknowledgement

This study was supported by TUBİTAK as VHAG 2003.

\section{References}

BELLAH JR 1997: How and when to perform lateral and vertical ear canal resection. Vet Med 92: 535-543

BODENHAMER RD, HUNTER JF, LUTTGEN PJ 1985: Brain stem auditory-evoked responses in the dog. Am J Vet Res 46: 1787-1792

COOK LB 2004: Neurologic evaluation of the ear. Vet Clin North Am-Small Anim Pract 34: 425-435

COX C 2002: Investigation of hearing loss in dogs. In Pract 24: 494-501

EGER CE, LINDSAY P 1997: Effects of otitis on hearing in dogs characterized by brainstem auditory evoked response testing. J Small Anim Pract 38: 380-386

KRAHWINKEL DJ, PARDO AD, SIMS MH, BUBB WJ 1993: Effect of total ablation of the external acoustic meatus and bulla osteotomy on auditory function in dogs. J Am Vet Med Assoc 202: 949-952

McANULTY JF, HATTEL A, HARVEY CE 1995: Wound healing and brain stem auditory evoked potentials after experimental ventral tympanic bulla osteotomy in dogs. Vet Surg 24: 9-14

SANDERS SG, BAGLEY RS 2003: Disorders of hearing and balance: The vestibulocochlear nerve (CN VIII) and associated structures. In: DEWEY CW (Ed.): A practical guide to canine and feline neurology. Iowa State Press, Iowa, pp. 213-240

SIMS MH 1988: Electrodiagnostic evaluation of auditory function. Vet Clin North Am Small Anim Pract 18: 913-941

SIMS HS 2003: Clinical evaluation of auditory function. In: SLATTER D (Ed.): Textbook of small animal surgery, $3^{\text {rd }}$ ed. WB Saunders, Philadelphia, pp. 1767-1773

STICKNEY M, REDDIN-PARKER S, McIAUGHLIN R 2004: Surgical treatment of chronic otitis externa in dogs. In: Symposium on otitis externa in dogs. Vet Med 99: 274-287 
Plate I

Besalti O. et al.: The Effect of Chronic ... pp. 615-624

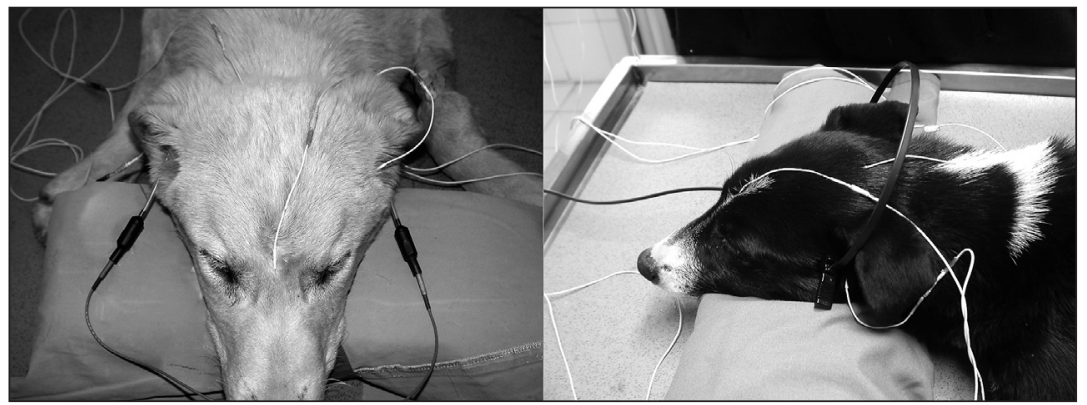

Fig. 1. Configuration of recording procedures for BAEP

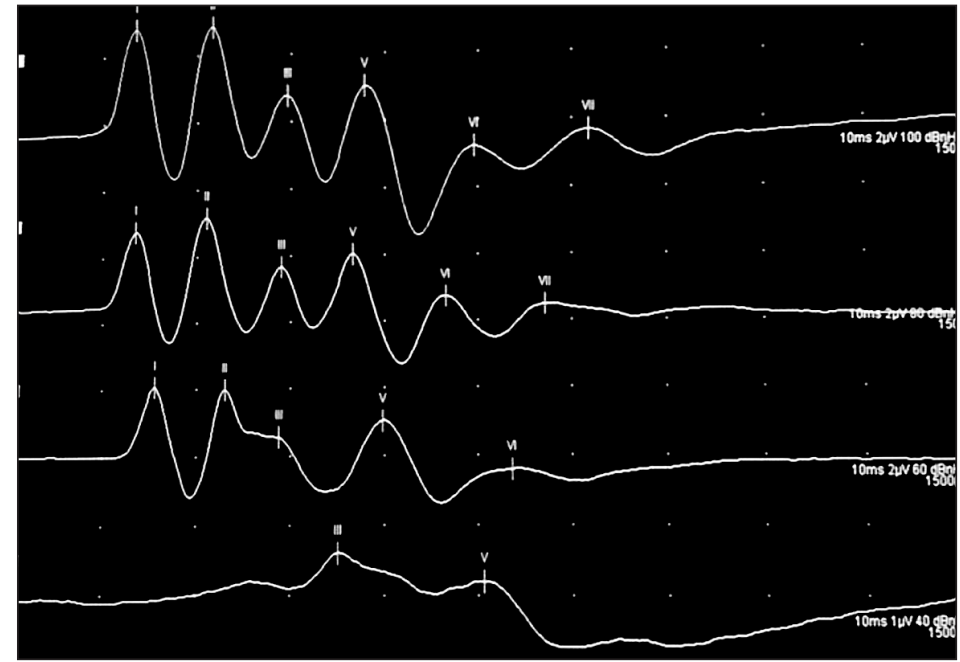

Fig. 2. BAEP at different stimulus intensities elicited by air clicks 
